\title{
A new branch-and-cut algorithm for the Generalized Directed Rural Postman Problem
}

\author{
Thais Ávila, Ángel Corberán \\ Dept. d'Estadística i Investigació Operativa, Universitat de València, Spain \\ Isaac Plana \\ Dept. de Matemáticas para la Economía y la Empresa, Universitat de València, Spain \\ José M. Sanchis \\ Dept. de Matemática Aplicada, Universidad Politécnica de Valencia, Spain
}

\begin{abstract}
The Generalized Directed Rural Postman Problem, also known as the Close-Enough Arc Routing Problem, is an arc routing problem with some interesting real-life applications, such as routing for meter reading. In this article we introduce two new formulations for this problem as well as various families of new valid inequalities that are used to design and implement a branch-and-cut algorithm. The computational results obtained on test bed instances from the literature show that this algorithm outperforms the existing exact methods.
\end{abstract}

Keywords: Generalized Rural Postman Problem, Close-Enough Arc Routing Problem, branch-and-cut.

\section{Introduction}

Let $G=(V, A)$ be a directed graph with set of vertices $V$ and set of $\operatorname{arcs} A$, and let $c_{i j} \geq 0$ be the cost associated with the traversal of arc $(i, j) \in A$. Given a family of arc subsets $\mathbb{H}=$ $\left\{H_{1}, \ldots, H_{L}\right\}$, the Generalized Directed Rural Postman Problem (GDRPP) consists of finding a minimum cost tour starting and ending at the depot and traversing at least one arc from each subset $H_{m}, m=1, \ldots, L$. These subsets $H_{m}$ do not need to be disjoint nor induce connected subgraphs.

This problem, without considering the presence of a depot, was introduced by Drexl in his doctoral thesis [7]. He noted that when each set $H_{m}$ consists of a single arc, the GDRPP reduces to the well known Directed Rural Postman Problem (DRPP) and therefore the GDRPP is NPhard. He proposed a formulation and a branch-and-cut algorithm producing good computational results. A more recent version of his work was published in [8].

However, it was Shuttleworth et al. [11] who presented this problem in the context of constructing routes for meter reading, calling it the Close-Enough Traveling Salesman Problem. In this application, a vehicle with a receiver travels through a series of neighborhoods. If the vehicle gets closer than a certain distance to a meter (customer), the receiver is able to record the gas, water, or electricity consumption. Therefore, the vehicle does not need to traverse every street, but only a few, in order to get close enough to each meter. The set of streets from which a certain meter $m$ can be read defines the set $H_{m}$. In their work, Shuttleworth et al. proposed four heuristics to solve eight real-life instances with an average of 900 customers and 9000 streets each. The procedures have essentially two phases: first a subset of streets to be traversed is 
selected, and then a route starting and ending at the depot and traversing all these streets is found. The first phase is obviously related to the resolution of a Set Covering Problem (SCP), while the second consists of solving a Directed Rural Postman Problem (DRPP) (or a Directed General Routing Problem if the depot is not incident with a selected street).

Note that in most arc routing problems each customer must be serviced from exactly one street, and thus the goal is to find a route traversing all the streets in a given set. However, in the GDRPP each customer can be serviced from one or more streets, so the vehicle only needs to traverse one of them (see Figure 1). The streets (arcs) from which a customer can be serviced form the set $H_{m}$ and the goal is to find a minimum cost tour traversing at least one arc from each $H_{m}$.

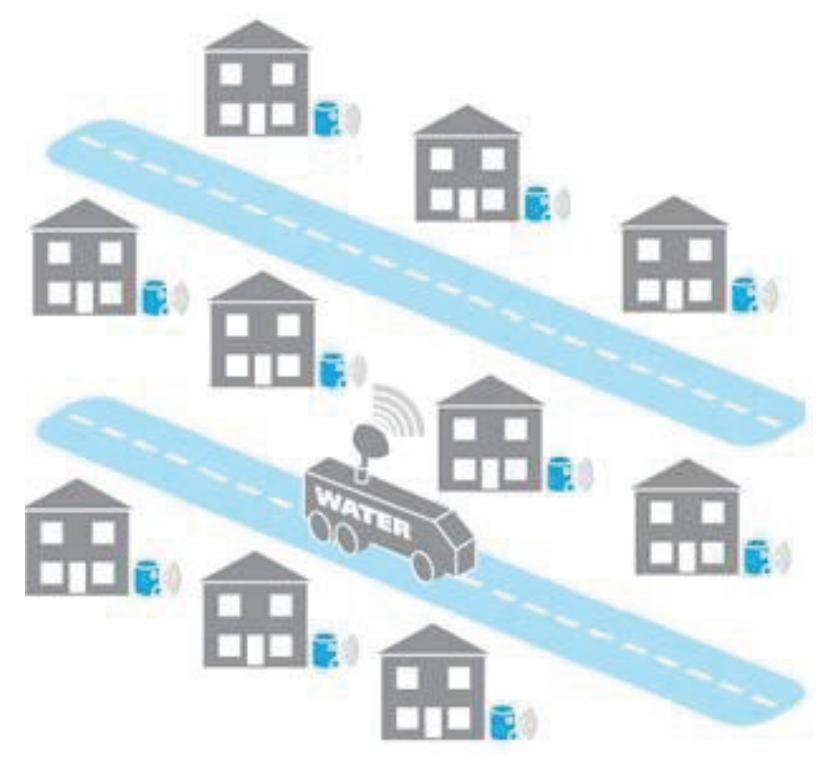

Figure 1: Remote reading of meters

More recently, Hà et al. [10] studied this problem, which they called the Close-Enough Arc Routing Problem, and proposed a new formulation, which they compared with a previous one introduced by the same authors [9] and the one by Drexl [7, 8]. Moreover, they proposed a branch-and-cut algorithm providing very good computational results on large-size instances.

In this work, we present two new mathematical formulations for the GDRPP and study the polyhedron of solutions associated with one of them. We also describe several new families of valid inequalities that are used in a new branch-and-cut algorithm. Finally, we compare the performance of our algorithm with that of the branch-and-cut by Hà et al. [10]. From the results obtained, we can conclude that our algorithm is, to our knowledge, the best existing exact method of solving this problem.

\section{Problem formulation}

We will denote by $A_{R}=H_{1} \cup H_{2} \cup \cdots \cup H_{L}$ (the set of arcs from which at least one customer can be serviced) and $A_{N R}=A \backslash A_{R}$. Given a subset of vertices $S \subset V$, we define $\delta^{+}(S)=\{(i, j) \in$ $A: \quad i \in S, \quad j \in V \backslash S\}, \delta^{-}(S)=\{(i, j) \in A: \quad i \in V \backslash S, \quad j \in S\}, \delta(S)=\delta^{+}(S) \cup \delta^{-}(S)$, $A(S)=\{(i, j) \in A: i, j \in S\}$, and $A_{R}(S)=\left\{(i, j) \in A_{R}: i, j \in S\right\}$. As usual, we will use $\delta^{+}(i)$ instead of $\delta^{+}(\{i\})$.

A closed walk starting and ending at the depot (vertex 1) and traversing at least one arc 
in each $H_{m}$ is called a GDRPP solution. In order to formulate the problem, we represent a GDRPP solution using two sets of variables:

- For each $\operatorname{arc}(i, j) \in A$, let $x_{i j}$ be the number of times arc $(i, j)$ is traversed.

- For each $\operatorname{arc}(i, j) \in A_{R}$, let $y_{i j}$ be equal to 1 if at least one customer is serviced from this arc, and 0 otherwise.

Although the problem can be formulated using only $x_{i j}$ variables (see Section 4 ), $y_{i j}$ variables are useful to express some valid inequalities and design the corresponding separation algorithms. The GDRPP can then be formulated as follows:

$$
\begin{aligned}
\text { Minimize } \sum_{(i, j) \in A} c_{i j} x_{i j} & \\
\text { s.t.: } x\left(\delta^{+}(i)\right)-x\left(\delta^{-}(i)\right)=0, & \forall i \in V \\
x\left(\delta^{+}(S)\right) \geq 1, & \forall S \subseteq V \backslash\{1\}: \exists H_{m} \subseteq A(S) \cup \delta(S) \\
x\left(\delta^{+}(S)\right) \geq y_{i j}, & \forall S \subset V \backslash\{1\}: \nexists H_{m} \subseteq A(S) \cup \delta(S), \forall(i, j) \in A_{R}(S) \\
\sum_{(i, j) \in H_{m}} y_{i j} \geq 1, & \forall H_{m} \in \mathbb{H} \\
x_{i j} \geq y_{i j}, & \forall(i, j) \in A_{R} \\
x_{i j} \geq 0, & \forall(i, j) \in A_{N R} \\
0 \leq y_{i j} \leq 1, & \forall(i, j) \in A_{R} \\
x_{i j} \text { integer, } & \forall(i, j) \in A \\
y_{i j} \text { integer, } & \forall(i, j) \in A_{R}
\end{aligned}
$$

Constraints (2) are the symmetry equations. Connectivity inequalities (3) and (4) guarantee that the optimal solution will be connected and connected to the depot. Service inequalities (5) guarantee that all the customers are serviced, while constraints (6) state that if a customer is serviced from an arc, this arc must be traversed. Constraints (7) to (10) are the nonnegativity and integrality inequalities.

We will call any vector $(x, y) \in \mathbb{R}^{\left|A_{|+|}\right| \mathbb{A}_{\mathbb{R}} \mid}$ satisfying (2) to (10) a GDRPP tour, or tour for short. Any GDRPP solution is a GDRPP tour, but given that this formulation allows the existence of subtours that are disconnected from the depot and do not service any customer, not all GDRPP tours are GDRPP solutions. Nevertheless, since all the costs $c_{i j}$ are non-negative, there will always be an optimal GDRPP tour not containing such subtours.

As mentioned in the introduction, the GDRPP can be considered a combination of two optimization problems, the Set Covering Problem (SCP) and the Directed General Routing Problem (DGRP):

- Given a set $E=\left\{e_{1}, \ldots, e_{n}\right\}$, a cost $c_{j} \geq 0$ associated with each element $e_{j} \in E$, and a family $\mathbb{S}=\left\{S_{1}, \ldots, S_{L}\right\}$ of subsets of $E$, the SCP consists of finding a minimum cost subset $E^{\prime} \subseteq E$ containing at least one element $e_{j}$ from each subset $S_{i}$.

- Given a directed graph $G=(V, A)$, with a cost $c_{i j}$ associated with each arc $a_{i j} \in A$, and given two subsets $A_{R} \subseteq A, V_{R} \subseteq V$ of required arcs and required vertices, respectively, the DGRP consists of finding a tour in $G$ with minimum cost traversing each required arc and visiting each required vertex at least once. 
Let us explain the relationship of the GDRPP with these two problems. Consider a tour for the GDRPP in graph $G=(V, A),(x, y) \in \mathbb{R}^{|A|+\left|A_{R}\right|}$, and let $A^{\prime}$ be the set of $\operatorname{arcs}(i, j) \in A_{R}$ such that $y_{i j}=1$. $A^{\prime}$ therefore defines a feasible solution for the SCP defined on the set $E=A_{R}$ and the family of subsets $\mathbb{S}=\mathbb{H}=\left\{H_{1}, \ldots, H_{L}\right\}$. Moreover, the vector $x$ represents a feasible solution for the DGRP in graph $G$ considering $A^{\prime}$ as required arcs and the depot as a required vertex. Conversely, we can find a tour $(x, y)$ for the GDRPP in $G$ by proceeding in two phases. First, we look for a feasible solution $A^{\prime}$ for the SCP defined by $E=A_{R}$ and $\mathbb{S}=\mathbb{H}$, and we define $y_{i j}=1$ for each arc $(i, j) \in A^{\prime}$. Then, from the SCP solution, we look for a feasible solution $x$ for the DGRP defined in the graph $G$ considering $A^{\prime}$ as the set of required arcs and the depot as the only required vertex. Obviously, even if both phases are solved optimally, the tour obtained for the GDRPP is not necessarily optimal.

Note that we can also build a tour for the GDRPP, $(x, y)$, by taking a tour $x$ for the DGRP defined in the graph $G$ using all the arcs in $H_{1} \cup \cdots \cup H_{L}$ as required arcs and the depot as the only required vertex, and a solution $y$ for the SCP defined with $E=H_{1} \cup \cdots \cup H_{L}$ and $\mathbb{S}=\mathbb{H}$. This kind of tour will be used in some of the proofs presented in this paper.

\section{The GDRPP polyhedron}

Let us define $\operatorname{GDRPP}(G)$ as the convex hull of all the tours for the GDRPP in $G$, i.e.

$$
\operatorname{GDRPP}(G)=\operatorname{Conv}\left\{(x, y) \in \mathbb{R}^{|\mathbb{A}|+\left|\mathbb{A}_{\mathbb{R}}\right|}:(x, y) \text { is a GDRPP tour }\right\} .
$$

It is not difficult to see that $\operatorname{GDRPP}(G)$ is an unbounded polyhedron.

\subsection{Basic properties}

Theorem 1 If $G$ is strongly connected, $\operatorname{dim}(\operatorname{GDRPP}(G))=|A|+\left|A_{R}\right|-|V|+1$ if and only if $\left|H_{m}\right| \geq 2 \forall H_{m} \in \mathbb{H}$.

Proof: Since exactly $|V|-1$ of the $|V|$ symmetry equations (2) are linearly independent, $\operatorname{dim}(\operatorname{GDRPP}(G)) \leq|A|+\left|A_{R}\right|-|V|+1$.

If there is a set containing a single arc, $H_{m}=\{(i, j)\}$, then $y_{i j}=1$ holds and $\operatorname{dim}(\operatorname{GDRPP}(G))<|A|+\left|A_{R}\right|-|V|+1$.

Conversely, let us suppose that $\left|H_{c}\right| \geq 2 \forall H_{m} \in \mathbb{H}$. Consider the DGRP on $G$ with all the $\operatorname{arcs}$ in $A_{R}$ as required arcs and the depot as a required vertex. Since $G$ is strongly connected, $\operatorname{dim}(\operatorname{DGRP}(G))=|A|-|V|+1$ (see [1]), and there are $k+1$ affinely independent DGRP tours $x^{1}, x^{2}, \ldots, x^{k+1}$, where $k=|A|-|V|+1$. Note that these tours traverse all the arcs in $A_{R}$ and visit the depot, and therefore they can be completed with vectors $y$ to define GDRPP tours $(x, y)$. Let us consider now the SCP with set $A_{R}$ and the family $\mathbb{H}$ of subsets. Given that $\left|H_{m}\right| \geq 2$, the dimension of the corresponding SCP polyhedron is $\left|A_{R}\right|$ (see Balas and $\mathrm{Ng}[2]$ ), and there are $m+1$ affinely independent SCP solutions $y^{1}, y^{2}, \ldots, y^{m+1}$, with $m=\left|A_{R}\right|$. Then $\left(x^{1}, y^{1}\right), \ldots,\left(x^{k+1}, y^{1}\right),\left(x^{1}, y^{2}\right), \ldots,\left(x^{1}, y^{m+1}\right)$ are $k+1+m$ affinely independent GDRPP tours and $\operatorname{dim}(\operatorname{GDRPP}(G)) \geq k+m=|A|+\left|A_{R}\right|-|V|+1$.

In what follows, we will assume that $\left|H_{m}\right| \geq 2$ for all $H_{m} \in \mathbb{H}$ and hence $\operatorname{dim}(\operatorname{GDRPP}(G))=|A|+\left|A_{R}\right|-|V|+1$. 
Now we will prove that, under certain conditions, some of the inequalities in the GDRPP formulation induce facets of the GDRPP polyhedron.

Theorem 2 Let $(i, j) \in A_{N R}$. If $G \backslash\{(i, j)\}$ is strongly connected, then inequality $x_{i j} \geq 0$ induces a facet of $\operatorname{GDRPP}(G)$.

Proof: Consider graph $G \backslash\{(i, j)\}$. Since $(i, j) \in A_{N R},\left|H_{m}\right| \geq 2$ still holds for every $H_{m} \in \mathbb{H}$, and we can find $x^{1}, \ldots, x^{k+1}$ affinely independent tours for the GDRPP defined on $G \backslash\{(i, j)\}$, where $k+1=\left(|A|-1+\left|A_{R}\right|-|V|+1\right)+1=|A|+\left|A_{R}\right|-|V|+1=\operatorname{dim}(\operatorname{GDRPP}(G))$. These tours are also tours for the GDRPP defined on $G$ and satisfy $x_{i j}=0$. Therefore $x_{i j} \geq 0$ induces a facet of $\operatorname{GDRPP}(G)$.

Theorem 3 Trivial inequalities $y_{i j} \leq 1,(i, j) \in A_{R}$, are facet-inducing for $\operatorname{GDRPP}(G)$.

Proof: It is well-known that inequality $y_{i j} \leq 1$ is facet-inducing for the SCP polyhedron (see Balas and $\mathrm{Ng}[2])$. Therefore, there are $m$ affinely independent solutions $y^{1}, \ldots, y^{m}, m=\left|A_{R}\right|$, satisfying $y_{i j}=1$.

Let us consider the DGRP defined on graph $G$ where all the arcs in $A_{R}$ are taken as required arcs and the depot is a required vertex. Since $G$ is strongly connected, $\operatorname{dim}(\operatorname{DGRP}(G))=|A|-$ $|V|+1=k$ and there are $k+1$ affinely independent DGRP tours in $G, x^{1}, x^{2}, \ldots, x^{k+1}$. Merging vectors $x$ and $y$, we obtain $k+1+m-1=|A|-|V|+1+\left|A_{R}\right|$ GDRPP tours, $\left(x^{1}, y^{1}\right), \ldots,\left(x^{k+1}, y^{1}\right),\left(x^{1}, y^{2}\right), \ldots,\left(x^{1}, y^{m}\right)$, satisfying $y_{i j}=1$. Hence inequality $y_{i j} \leq 1$ is facet-inducing for $\operatorname{GDRPP}(G)$.

Theorem 4 Trivial inequalities $y_{i j} \geq 0,(i, j) \in A_{R}$, are facet-inducing for $\operatorname{GDRPP}(G)$ if $\left|H_{m}\right| \geq 3$, for all $H_{m} \in \mathbb{H}$ such that $(i, j) \in H_{m}$.

Proof: The proof is similar to that of Theorem 3.

Theorem 5 Let $(i, j) \in A_{R}$. If $G \backslash\{(i, j)\}$ is strongly connected and $\left|H_{m}\right| \geq 3$, for all $H_{m} \in \mathbb{H}$ such that $(i, j) \in H_{m}$, inequalities $x_{i j} \geq y_{i j}$ are facet-inducing for $\operatorname{GDRPP}(G)$.

Proof: Since $G \backslash\{(i, j)\}$ is strongly connected and $\left|H_{m} \backslash\{(i, j)\}\right| \geq 2, \forall H_{m}$, there are $\left(x^{1}, y^{1}\right), \ldots,\left(x^{k+1}, y^{k+1}\right), k=(|A|-1)+\left(\left|A_{R}\right|-1\right)-|V|+1=|A|+\left|A_{R}\right|-|V|-1$, affinely independent GDRPP tours satisfying $x_{i j}=0$ and $y_{i j}=0$, and therefore $x_{i j}=y_{i j}$. Given that there is a path $P_{j i}$ in $G$ from $j$ to $i$, we can obtain a new tour $(\bar{x}, \bar{y})$ satisfying $\bar{x}_{i j}=\bar{y}_{i j}=1$ by adding the $\operatorname{arcs}$ of $P_{j i}$ plus arc $(i, j)$ to $x^{1}$ and setting $\bar{y}_{i j}=1$. Then we have $k+2=|A|+\left|A_{R}\right|-|V|+1$ tours for the GDRPP satisfying $x_{i j}=y_{i j}$. As shown in the matrix in Figure 2, these vectors are affinely independent and the inequality is facet-inducing.

\begin{tabular}{|c|c|c|}
$A$ & $A_{R} \backslash\{(i, j)\}$ & $(i, j)$ \\
\hline$x^{1}$ & $y^{1}$ & \\
$\vdots$ & $\vdots$ & 0 \\
$x^{k+1}$ & $y^{k+1}$ & \\
\hline $\bar{x}$ & $\bar{y}$ & 1 \\
\hline
\end{tabular}

Figure 2: Matrix appearing in the proof of Theorem 5 
Theorem 6 Inequalities $(5) \sum_{(i, j) \in H_{m}} y_{i j} \geq 1, \forall H_{m} \in \mathbb{H}$, are facet-inducing for $\operatorname{GDRPP}(G)$ if

- there is no $H_{q} \subset H_{m}$, and

- for all $\left(i^{\prime}, j^{\prime}\right) \in A_{R} \backslash H_{m}$, there is an arc $(i, j) \in H_{m}$ such that $(i, j) \in H_{q} \forall H_{q}$ such that $H_{q} \backslash H_{m}=\left\{\left(i^{\prime}, j^{\prime}\right)\right\}$.

Proof: It is known that inequality $\sum_{(i, j) \in H_{m}} y_{i j} \geq 1$ is facet-inducing for the SCP polyhedron if and only if the two conditions above hold (see Balas and $\mathrm{Ng}[2]$ ). Hence, there are $m=\left|A_{R}\right|$ affinely independent solutions $y^{1}, \ldots, y^{m}$ for the SCP satisfying $\sum_{(i, j) \in H_{m}} y_{i j}=1$. Let us consider now the DGRP defined on $G$, taking all the arcs in $A_{R}$ as required and the depot as a required vertex. Since $\operatorname{dim}(\operatorname{DGRP}(G))=|A|-|V|+1=k$, there are $k+1$ affinely independent tours for the DGRP, $x^{1}, x^{2}, \ldots, x^{k+1}$. Merging vectors $x$ and $y$, we obtain $k+1+m-1=|A|-|V|+1+\left|A_{R}\right|$ tours for the GDRPP, $\left(x^{1}, y^{1}\right), \ldots,\left(x^{k+1}, y^{1}\right),\left(x^{1}, y^{2}\right), \ldots,\left(x^{1}, y^{m}\right)$, satisfying $\sum_{(i, j) \in H_{m}} y_{i j}=1$. Therefore, inequality $\sum_{(i, j) \in H_{m}} y_{i j} \geq 1$ is facet-inducing for $\operatorname{GDRPP}(G)$.

\subsection{Connectivity inequalities}

In this section we study the connectivity inequalities present at the formulation, as well as an interesting generalization of them.

We believe that connectivity inequalities (4), $x\left(\delta^{+}(S)\right) \geq y_{i j}$, are facet-inducing for $\operatorname{GDRPP}(G)$ if $G$ and $G(V \backslash S)$ are strongly connected and $\left|H_{q} \backslash \delta(S)\right| \geq 2 \forall H_{q} \in \mathbb{H}$, but we have not been able to prove it. The following theorem proves that connectivity inequalities $(3), x\left(\delta^{+}(S)\right) \geq 1$, are facet-inducing for $\operatorname{GDRPP}(G)$.

Theorem 7 Connectivity inequalities (3), $x\left(\delta^{+}(S)\right) \geq 1, \forall S \subseteq V \backslash\{1\}$ such that $\exists H_{m} \subseteq$ $A(S) \cup \delta(S)$, are facet-inducing for $\operatorname{GDRPP}(G)$ if $G(S)$ and $G(V \backslash S)$ are strongly connected and $\left|H_{q} \backslash \delta(S)\right| \geq 2 \forall H_{q} \in \mathbb{H}$.

Proof: Let us consider the DGRP defined on $G$, taking $A_{R}^{\prime}=A_{R} \backslash \delta(S)$ as the required arcs and the depot as a required vertex. Since $G(S)$ and $G(V \backslash S)$ are strongly connected, the inequality $x\left(\delta^{+}(S)\right) \geq 1$ is facet-inducing for the polyhedron associated with the DGRP (see Ávila et al. [1]) and there are $k=|A|-|V|+1$ affinely independent tours, $x^{1}, \ldots, x^{k}$, for the DGRP traversing all the arcs in $A_{R}^{\prime}$ and satisfying $x\left(\delta^{+}(S)\right)=1$. Moreover, given that $\left|H_{q} \backslash \delta(S)\right| \geq 2$ $\forall H_{q} \in \mathbb{H}$, the polyhedron associated with the SCP defined on the ground set $A_{R}^{\prime}$ is of full dimension, and there are $m+1=\left|A_{R}^{\prime}\right|+1$ affinely independent SCP solutions $y^{1}, \ldots, y^{m+1}$. We extend these vectors $y^{r}$ by adding zeros in the components associated with the arcs in $\delta_{R}(S)$ to obtain vectors $\bar{y}^{r}$. Merging these last vectors with vectors $x$, we get $k+m=|A|-|V|+\left|A_{R}^{\prime}\right|+1$ tours for the GDRPP $,\left(x^{1}, \bar{y}^{1}\right), \ldots,\left(x^{k}, \bar{y}^{1}\right),\left(x^{1}, \bar{y}^{2}\right), \ldots,\left(x^{1}, \bar{y}^{m+1}\right)$ satisfying $x\left(\delta^{+}(S)\right)=1$.

For each arc $\left(i_{0}, j_{0}\right) \in \delta_{R}(S)$, since $x\left(\delta^{+}(S)\right) \geq 1$ is facet-inducing for the DGRP polyhedron, it can be seen that there is a DGRP tour $x^{*}$ traversing $\left(i_{0}, j_{0}\right)$ and satisfying $x\left(\delta^{+}(S)\right)=1$. Therefore, we can define a new GDRPP tour $\left(x^{*}, y^{*}\right)$, where $y^{*}$ is obtained from $\bar{y}^{1}$ by replacing $y_{i_{0}, j_{0}}^{*}=1$.

If we express all these GDRPP tours as rows in a matrix, we obtain the matrix shown in Figure 3, which is of full rank. Hence inequalities (3) are facet-inducing. 


\begin{tabular}{|c|c|c|}
$A$ & $A_{R}^{\prime}$ & $\delta_{R}(S)$ \\
\hline$x^{1}$ & $y^{1}$ & \\
$\vdots$ & $\vdots$ & 0 \\
$x^{k}$ & $y^{1}$ & \\
\hline$x^{1}$ & $y^{2}$ & \\
$\vdots$ & $\vdots$ & 0 \\
$x^{1}$ & $y^{m+1}$ & \\
\hline & $y^{1}$ & $\mathrm{I}$ \\
$*$ & $y^{1}$ & $\mathrm{I}$ \\
\hline
\end{tabular}

Figure 3: Matrix appearing in the proof of Theorem 7

Connectivity inequalities $(3), x\left(\delta^{+}(S)\right) \geq 1$, are a special case of the following more general family of inequalities:

$$
x\left(\delta^{+}(S)\right) \geq 1-y\left(H_{m} \cap A_{R}(V \backslash S)\right), \quad \forall S \subset V \backslash\{1\} \quad \forall H_{m} \in \mathbb{H}
$$

For example, consider a subset of vertices $S$ and a subset $H_{m}$ such as those depicted in Figure 4, where $H_{m} \cap A_{R}(V \backslash S)=\{a\}$. The corresponding connectivity inequality (11) is $x\left(\delta^{+}(S)\right) \geq 1-y_{a}$. Note also that the above inequality reduces to inequality (3) when there is an $H_{m} \subseteq A(S) \cup \delta(S)$.

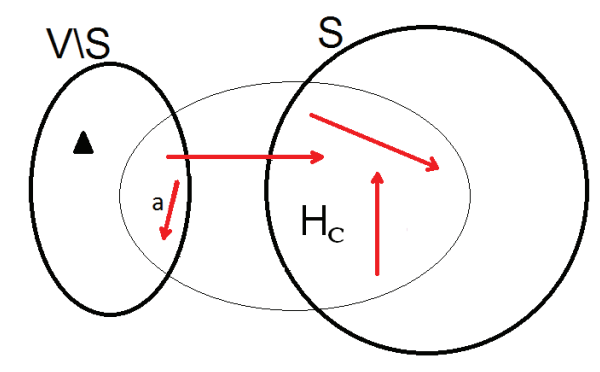

Figure 4: Structure of the connectivity inequality $x\left(\delta^{+}(S)\right) \geq 1-y_{a}$

Theorem 8 Connectivity inequalities (11), $x\left(\delta^{+}(S)\right) \geq 1-y\left(H_{m} \cap A_{R}(V \backslash S)\right), \forall S \subset V \backslash\{1\}$, $\forall H_{m} \in \mathbb{H}$, are valid for the GDRPP.

Proof: Let $\left(x^{*}, y^{*}\right)$ be a tour for the GDRPP. If $y^{*}\left(H_{m} \cap A_{R}(V \backslash S)\right)=0$, then this tour must service at least one arc in $H_{m} \cap(A(S) \cup \delta(S))$. In order to do this, it has to traverse $\delta(S)$, and so $x^{*}\left(\delta^{+}(S)\right) \geq 1$ holds. If $y^{*}\left(H_{m} \cap A_{R}(V \backslash S)\right) \geq 1$, then the inequality is obviously satisfied.

Theorem 9 Connectivity inequalities (11), $x\left(\delta^{+}(S)\right) \geq 1-y\left(H_{m} \cap A_{R}(V \backslash S)\right), \forall S \subset V \backslash\{1\}$ and $\forall H_{m} \in \mathbb{H}$, are facet-inducing for $\operatorname{GDRPP}(G)$ if $G(S)$ and $G(V \backslash S)$ are strongly connected, $\left|H_{q} \backslash\left(\delta(S) \cup\left(H_{m} \cap A_{R}(V \backslash S)\right)\right)\right| \geq 2 \forall H_{q} \in \mathbb{H}$, and, in the case in which $H_{m} \cap A_{R}(V \backslash S) \neq \emptyset$, the following conditions are satisfied:

(1) $\forall H_{q} \in \mathbb{H} \quad H_{q} \cap A_{R}(V \backslash S) \neq \emptyset$ and

(2) $\nexists H_{q} \in \mathbb{H}$ such that $H_{q} \cap A_{R}(V \backslash S) \varsubsetneqq H_{m} \cap A_{R}(V \backslash S)$. 
Proof: If $H_{m} \cap A_{R}(V \backslash S)=\emptyset$, i.e. $H_{m} \subseteq \delta(S) \cup A(S)$, then we have the connectivity inequality (3), $x\left(\delta^{+}(S)\right) \geq 1$, which has been proved to be facet-inducing in Theorem 7 .

Let us suppose now that $H_{m} \cap A_{R}(V \backslash S) \neq \emptyset$. Consider the DGRP defined on $G$, taking $A_{R}^{\prime}=A_{R} \backslash\left(\delta(S) \cup\left(H_{m} \cap A_{R}(V \backslash S)\right)\right)$ as the required arcs and the depot as a required vertex. Since $G(S)$ and $G(V \backslash S)$ are strongly connected, the inequality $x\left(\delta^{+}(S)\right) \geq 1$ is facet-inducing for the polyhedron associated with the DGRP (see Ávila et al. [1]) and there are $k=|A|-|V|+1$ affinely independent tours, $x^{1}, \ldots, x^{k}$, for the DGRP traversing all the arcs in $A_{R}^{\prime}$ and satisfying $x\left(\delta^{+}(S)\right)=1$. Moreover, given that $\left|H_{q} \backslash\left(\delta(S) \cup\left(H_{m} \cap A_{R}(V \backslash S)\right)\right)\right| \geq 2 \forall H_{q}$, the polyhedron associated with the SCP defined on the ground set $A_{R}^{\prime}$ is full-dimensional, and there are $m+1=$ $\left|A_{R}^{\prime}\right|+1$ affinely independent SCP solutions $y^{1}, \ldots, y^{m+1}$. We extend these vectors $y^{r}$ by adding zeros in the components associated with the arcs in $\delta_{R}(S) \cup\left(H_{m} \cap A_{R}(V \backslash S)\right.$ to obtain vectors $\bar{y}^{r}$. Merging these last vectors with vectors $x$, we get $k+m=|A|-|V|+\left|A_{R}^{\prime}\right|+1$ tours for the GDRPP, $\left(x^{1}, \bar{y}^{1}\right), \ldots,\left(x^{k}, \bar{y}^{1}\right),\left(x^{1}, \bar{y}^{2}\right), \ldots,\left(x^{1}, \bar{y}^{m+1}\right)$, satisfying $x\left(\delta^{+}(S)\right)=1$ and $y\left(H_{m} \cap A_{R}(V \backslash S)=0\right.$.

For each arc $\left(i_{0}, j_{0}\right) \in \delta_{R}(S)$, since $x\left(\delta^{+}(S)\right) \geq 1$ is facet-inducing for the DGRP polyhedron, there is a DGRP tour $x^{*}$ traversing $\left(i_{0}, j_{0}\right)$ and satisfying $x\left(\delta^{+}(S)\right)=1$. Therefore, we can define a new GDRPP tour $\left(x^{*}, y^{*}\right)$, where $y^{*}$ is obtained from $\bar{y}^{1}$ by replacing $y_{i_{0}, j_{0}}^{*}=1$.

Finally, for each arc $\left(i_{0}, j_{0}\right) \in H_{m} \cap A_{R}(V \backslash S)$ we can define a GDRPP tour $(\widetilde{x}, \widetilde{y})$ satisfying $x\left(\delta^{+}(S)\right)=0$ (note that, since condition (1) is satisfied, all the customers can be serviced from arcs in $A(V \backslash S)$ ), and such that $\widetilde{y}_{i_{0} j_{0}}=1, \widetilde{y}_{i j}=0, \forall(i, j) \in H_{m} \cap A_{R}(V \backslash S) \backslash\left\{\left(i_{0}, j_{0}\right)\right\}$ (since condition (2) is satisfied, all the customers serviced from arcs in $H_{m} \cap A_{R}(V \backslash S) \backslash\left\{\left(i_{0}, j_{0}\right)\right\}$ can be also serviced from arcs in $\left.A_{R}(V \backslash S)\right)$. Therefore, $\widetilde{y}$ satisfies $y\left(H_{m} \cap A_{R}(V \backslash S)\right)=1$ and the tour $(\widetilde{x}, \widetilde{y})$ satisfies the inequality as an equality.

If we express these $|A|+\left|A_{R}\right|-|V|+1$ GDRPP tours as rows in a matrix, we obtain the matrix shown in Figure 5, which has full rank. Hence the inequality is facet-inducing.

\begin{tabular}{|c|c|c|c|}
$A$ & $A_{R}^{\prime}$ & $\delta_{R}(S)$ & $H_{m} \cap A_{R}(V \backslash S)$ \\
\hline$x^{1}$ & $y^{1}$ & & \\
$\vdots$ & $\vdots$ & 0 & 0 \\
$x^{k}$ & $y^{1}$ & & \\
\hline$x^{1}$ & $y^{2}$ & & 0 \\
$\vdots$ & $\vdots$ & 0 & \\
$x^{1}$ & $y^{m+1}$ & & 0 \\
$*$ & $y^{1}$ & $\mathrm{I}$ & \\
\hline$*$ & $y^{1}$ & & $\mathrm{I}$ \\
\hline
\end{tabular}

Figure 5: Matrix appearing in the proof of Theorem 9

\subsection{Parity inequalities}

Parity inequalities are a class of widely used inequalities in arc routing problems. They rely on the fact that a tour crosses any cutset an even (or zero) number of times. Although in most arc routing problems defined on directed graphs parity inequalities are implied by the symmetry equations, the new parity inequalities we propose here, which are related not only to the arcs in 
the cutset but also to some sets $H_{m}$, are not implied by the symmetry equations.

Let $S \subset V, F \subseteq \delta_{R}(S)$, and $F^{H}=\left\{H_{n_{1}}, H_{n_{2}}, \ldots, H_{n_{q}}\right\}$, with $|F|+q$ odd and $q>0$, such that (see Figure 6):

- $H_{n_{i}} \cap \delta(S) \neq \emptyset \quad \forall n_{i}$,

- $H_{n_{i}} \cap H_{n_{j}} \cap \delta(S)=\emptyset \quad \forall n_{i}, n_{j}$, and

- $F \cap H_{n_{i}}=\emptyset \forall n_{i}$.

The parity inequality (associated with $S, F$, and $F^{H}$ ) is

$$
x(\delta(S)) \geq 2 y(F)-|F|+\sum_{i=1}^{q}\left(1-2 y\left(H_{n_{i}} \backslash \delta(S)\right)\right)+1
$$

Note that for each subset $H_{n_{i}}$ such that $H_{n_{i}} \subseteq \delta(S)$, the corresponding term $1-2 y\left(H_{n_{i}} \backslash \delta(S)\right)$ in the RHS of (12) is 1 . In particular, when this happens with all the subsets $H_{n_{i}} \in F^{H}$, the inequality reduces to

$$
x(\delta(S)) \geq 2 y(F)-|F|+q+1,
$$

and if, in addition, $F=\emptyset$, the inequality becomes

$$
x(\delta(S)) \geq q+1 .
$$

Note also that, since $|F|+q$ is odd, inequality (12) can be written in the following more sparse way:

$$
x\left(\delta^{+}(S)\right) \geq y(F)+\frac{q+1-|F|}{2}-\sum_{i=1}^{q} y\left(H_{n_{i}} \backslash \delta(S)\right)
$$

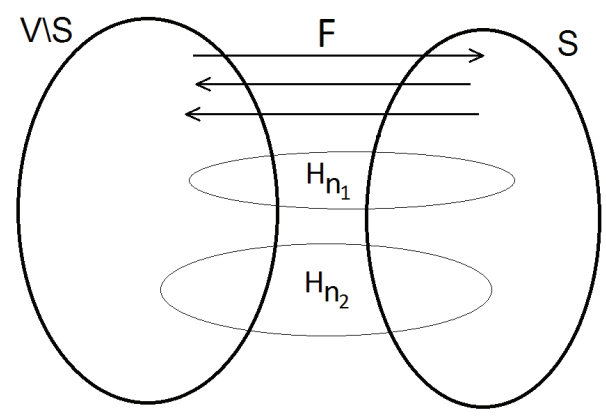

Figure 6: New parity constraints structure

Theorem 10 Parity inequalities (12) are valid for $\operatorname{GDRPP}(G)$.

Proof: Let $(x, y)$ be a GDRPP tour. We consider the following cases:

- $(x, y)$ services all the arcs in $F$ and does not service any arc in any of the sets $H_{n_{i}} \backslash \delta(S)$. In this case, the RHS of the inequality is $|F|+q+1$. Given that any tour must service at least one arc in each $H_{n_{i}},(x, y)$ services at least one arc in each $H_{n_{i}} \cap \delta(S), i=1 \ldots, q$. Therefore it traverses $\delta(S)$ at least $|F|+q$ times, and since $|F|+q$ is odd, the tour traverses $\delta(S)$ at least $|F|+q+1$ times, satisfying $x(\delta(S)) \geq|F|+q+1$. 
- $(x, y)$ services all but one of the arcs in $F$ and does not service any arc in any of the sets $H_{n_{i}} \backslash \delta(S)$. In this case, the RHS of the inequality is $|F|+q-1$. Given that the tour traverses and services at least one arc in each $H_{n_{i}}, i=1 \ldots, q$, and $|F|-1$ arcs in $F$, it traverses $\delta(S)$ at least $|F|+q-1$ times.

- $(x, y)$ services all the arcs in $F$ and exactly one arc in one of the sets $H_{n_{i}} \backslash \delta(S)$. In this case, the tour traverses at least $|F|+q-1 \operatorname{arcs}$ in $\delta(S)$ and the RHS of the inequality is also $|F|+q-1$.

- $(x, y)$ services all but one of the $\operatorname{arcs}$ in $F$ and exactly one arc in one of the sets $H_{n_{i}} \backslash \delta(S)$. In this case, the RHS of the inequality is $|F|+q-3$ and the tour traverses at least $|F|-1+q-1$ arcs in $\delta(S)$. Therefore, $x(\delta(S)) \geq|F|+q-2 \geq|F|+q-3$.

- In any other case, a similar argument proves that the inequality is satisfied.

\section{$3.4 \quad \mathrm{~K}-\mathrm{C}$ inequalities}

K-C inequalities were introduced by Corberán and Sanchis [6] for the undirected RPP and have been adapted to many other arc routing problems. In this section we present a generalization of these inequalities for the GDRPP.

Consider a partition of vertex set $V$ into $K+1$ subsets $\left\{M_{0} \cup M_{K}, M_{1}, \ldots, M_{K-1}\right\}$, with $K \geq 3$. Let $\left\{I_{1}, I_{2}\right\}$ be a partition of the set $\{1,2, \ldots, K-1\}$ such that (see Figure 7 )

- for each $j \in I_{1}$, either $1 \in M_{j}$ or there is an $H_{m_{j}}$ such that $H_{m_{j}} \cap A_{R}\left(M_{j}\right) \neq \emptyset$,

- for each $j \in I_{2}$, there is a required $\operatorname{arc} a^{j} \in A_{R}\left(M_{j}\right)$, and

- the induced subgraphs $G\left(M_{0}\right), G\left(M_{1}\right), \ldots, G\left(M_{K}\right)$ are strongly connected.

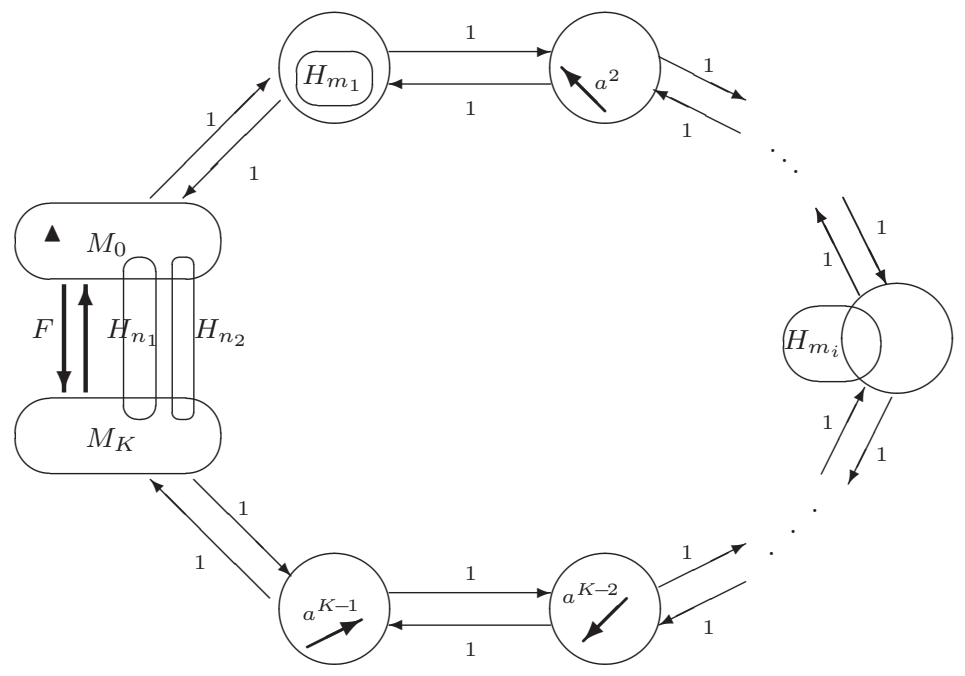

Figure 7: $K$-C inequality structure

For simplicity, we denote $A_{R}^{O K}=\left(M_{0}: M_{K}\right)_{R} \cup\left(M_{K}: M_{0}\right)_{R}$. Let $F \subseteq A_{R}^{O K}$ be a set of arcs and let $F^{H}=\left\{H_{n_{1}}, H_{n_{2}}, \ldots, H_{n_{q}}\right\}$ a set of customers satisfying

- $|F|+q$ is even, 
- $H_{n_{i}} \cap A_{R}^{O K} \neq \emptyset \quad \forall i$,

- $H_{n_{i}} \cap H_{n_{j}} \cap A_{R}^{O K}=\emptyset \quad \forall i, \forall j$, and

- $F \cap H_{n_{i}}=\emptyset \quad \forall i$.

We assume that if the depot is not in a set $M_{j}, j \in I_{1}$, then $1 \in M_{0}$. The following inequality is called a $\mathrm{K}-\mathrm{C}$ inequality for the GDRPP:

$$
\begin{gathered}
(K-2)\left(x\left(M_{0}: M_{K}\right)+x\left(M_{K}: M_{0}\right)\right)+\sum_{\substack{0 \leq i, j \leq K \\
(i, j) \neq(0, K)}}|i-j| x\left(M_{i}: M_{j}\right) \\
\geq(K-2)(2 y(F)-|F|)+(K-2) \sum_{i=1}^{q}\left(1-2 y\left(H_{n_{i}} \backslash A_{R}^{O K}\right)\right) \\
+2 \sum_{j \in I_{1}}\left(1-y\left(H_{m_{j}} \backslash A\left(M_{j}\right)\right)\right)+2 \sum_{j \in I_{2}} y_{a^{j}}
\end{gathered}
$$

In the GDRPP, unlike other arc routing problems, not all the arcs in $F$ have to be traversed, since the customers serviced by these arcs can also be serviced from other arcs. Similarly, it is not necessary to traverse arcs in each $H_{n_{i}} \cap A_{R}^{O K}$, as customers $H_{n_{i}}$ can be serviced from arcs in $H_{n_{i}} \backslash A_{R}^{O K}$, if such arcs exist. If they do not exist, i.e. $H_{n_{i}} \subseteq A_{R}^{O K}$, then it is necessary to traverse at least one arc in $H_{n_{i}}$. Hence, if $F=\emptyset$ and $H_{n_{i}} \subseteq A_{R}^{O K}$ for all $i=1, \ldots, q$, then all the GDRPP tours have to traverse at least $q \operatorname{arcs}$ in $A_{R}^{O K}$. Note that, in this case, the corresponding term in the RHS of the inequality (14) is $(K-2) q$. Moreover, in the GDRPP it is not necessary to visit all the subgraphs $G\left(M_{0} \cup M_{K}\right), G\left(M_{1}\right), \ldots, G\left(M_{K-1}\right)$, but only those $G\left(M_{j}\right), j \in I_{1}$, containing the depot or satisfying $H_{m_{j}} \subseteq A_{R}\left(M_{j}\right)$. Note that if $I_{2}=\emptyset$ and for each $j \in I_{1}$, either $1 \in M_{j}$ or $H_{m_{j}} \subseteq A_{R}\left(M_{j}\right)$, then the corresponding term in the RHS of the inequality (14) takes value $2(K-1)$. Therefore, the $\mathrm{K}-\mathrm{C}$ inequality for the GDRPP can be considered a generalization of the standard K-C inequality for the DGRP.

Theorem $11 K$-C inequalities (14) are valid for the GDRPP.

Proof: Let us call $\mathrm{KC}(x)$ to the left-hand side of the $\mathrm{K}-\mathrm{C}$ inequality (14) and let us denote by $c_{0}=(K-2)(|F|+q)+2(K-1)$, which is the right-hand side of a standard K-C inequality for the DGRP associated with an even number $|F|+q$ of required $\operatorname{arcs}$ between $M_{0}$ and $M_{K}$. Let $(x, y)$ be a GDRPP tour. We distinguish the following possibilities:

- $(x, y)$ services all the $\operatorname{arcs}$ in $F$ and all the $\operatorname{arcs} a^{j}, j \in I_{2}$, but does not traverse any arc in any of the sets $H_{n_{i}} \backslash A_{O K}^{R}$ or $H_{m_{j}} \backslash A_{R}\left(M_{j}\right)$. In this case, the RHS of (14) takes value $(K-2)|F|+(K-2) q+2\left|I_{1}\right|+2\left|I_{2}\right|=(K-2)(|F|+q)+2(K-1)=c_{0}$. Note that, since $x$ does not traverse any arc in $H_{n_{i}} \backslash A_{O K}^{R}$, then $(x, y)$ must service at least one arc in $H_{n_{i}} \cap A_{O K}^{R}$. The same holds for the sets $H_{m_{j}} \backslash A_{R}\left(M_{j}\right)$. Therefore, we have a $\mathrm{K}$-C structure and a tour $x$ traversing an even number $|F|+q$ of arcs between $M_{0}$ and $M_{K}$ and visiting all the subgraphs $G\left(M_{0} \cup M_{K}\right), G\left(M_{1}\right), \ldots, G\left(M_{K-1}\right)$. Since the standard K-C inequality is valid for the DGRP, $x$ will satisfy $\mathrm{KC}(x) \geq c_{0}$ and $(x, y)$ satisfies the $\mathrm{K}$-C inequality (14).

- $(x, y)$ services all but one of the arcs in $F$ and all the $\operatorname{arcs} a^{j}, j \in I_{2}$, and does not traverse any arc in any of the sets $H_{n_{i}} \backslash A_{O K}^{R}$ or $H_{m_{j}} \backslash A_{R}\left(M_{j}\right)$. In this case, the RHS of (14) takes value $(K-2)(|F|+q-2)+2(K-1)=c_{0}-2(K-2)$. The tour $(x, y)$ traverses $|F|+q-1$ arcs between $M_{0}$ and $M_{K}$ with cost $(K-2)(|F|+q-1)$. Since $|F|+q-1$ is odd, the tour can visit all the subgraphs $G\left(M_{0} \cup M_{K}\right), G\left(M_{1}\right), \ldots, G\left(M_{K-1}\right)$ with a path $G\left(M_{0}\right), G\left(M_{1}\right), \ldots, G\left(M_{K-1}\right), G\left(M_{K}\right), G\left(M_{0}\right)$, which has cost $K$. Therefore, $x$ satisfies $\mathrm{KC}(x) \geq(K-2)(|F|+q-1)+K=c_{0}-2(K-2)$ and $(x, y)$ satisfies (14). 
- $(x, y)$ services all the $\operatorname{arcs}$ in $F$ and all the $\operatorname{arcs} a^{j}, j \in I_{2}$, it services exactly one arc in one set $H_{n_{i}} \backslash A_{O K}^{R}$ and does not traverse any arc in any set $H_{m_{j}} \backslash A_{R}\left(M_{j}\right)$. In this case, the RHS of (14) takes value $(K-2)|F|+(K-2)(q-2)+2\left|I_{1}\right|+2\left|I_{2}\right|=c_{0}-2(K-2)$. As in the previous case, $(x, y)$ traverses an odd number $|F|+q-1$ of $\operatorname{arcs}$ between $M_{0}$ and $M_{K}$ and visits all the subgraphs $G\left(M_{0} \cup M_{K}\right), G\left(M_{1}\right), \ldots, G\left(M_{K-1}\right)$. Therefore, $\mathrm{KC}(x) \geq(K-2)(|F|+q-1)+K=c_{0}-2(K-2)$ and $(x, y)$ satisfies $(14)$.

- $(x, y)$ services all the $\operatorname{arcs}$ in $F$ and all but one of the $\operatorname{arcs} a^{j}, j \in I_{2}$, and does not traverse any arc in any of the sets $H_{n_{i}} \backslash A_{O K}^{R}$ or $H_{m_{j}} \backslash A_{R}\left(M_{j}\right)$. In this case, the RHS of (14) takes value $(K-2)|F|+(K-2) q+2\left|I_{1}\right|+2\left|I_{2}-1\right|=c_{0}-2$. We have a $\mathrm{K}-\mathrm{C}$ structure and $x$ traverses an even number $|F|+q$ of arcs between $M_{0}$ and $M_{K}$ and visits all but one of the subgraphs $G\left(M_{0} \cup M_{K}\right), G\left(M_{1}\right), \ldots, G\left(M_{K-1}\right)$. If $x$ had a cost lower than $c_{0}-2$, we could add two arcs with cost 1 to connect the remaining subgraph and obtain a DGRP tour in the K-C structure with cost less than $c_{0}$, which is impossible. Hence, $x$ satisfies $F(x) \geq c_{0}-2$ and $(x, y)$ satisfies inequality (14).

- $(x, y)$ services all the $\operatorname{arcs}$ in $F$ and all the $\operatorname{arcs} a^{j}, j \in I_{2}$, it does not traverse any arc in any of the sets $H_{n_{i}} \backslash A_{O K}^{R}$, and it services exactly one arc in one set $H_{m_{j}} \backslash A_{R}\left(M_{j}\right)$. In this case, the RHS of (14) takes value $(K-2)|F|+(K-2) q+2\left|I_{1}-1\right|+2\left|I_{2}\right|=c_{0}-2$. As in the previous case, $x$ traverses an even number $|F|+q$ of $\operatorname{arcs}$ between $M_{0}$ and $M_{K}$ and visits all but one of the subgraphs $G\left(M_{0} \cup M_{K}\right), G\left(M_{1}\right), \ldots, G\left(M_{K-1}\right)$. Using the same argument as before, we obtain $\mathrm{KC}(x) \geq c_{0}-2$, and therefore $(x, y)$ satisfies (14).

- In any other case, a similar argument proves the result.

\subsection{Dominance inequalities}

As mentioned in Section 2, the GDRPP is closely related to the Set Covering Problem (SCP). Many of the constraints known for the SCP can be used to strengthen the formulation for the GDRPP. In this section, we describe a class of inequalities used by Hà et al. [10] that will be included in our branch-and-cut algorithm.

Given two arcs $\left(i_{1}, j_{1}\right),\left(i_{2}, j_{2}\right) \in A_{R}$, the first is said to dominate the second if for any set $H_{m} \in \mathbb{H}$ such that $\left(i_{2}, j_{2}\right) \in H_{m}$ then $\left(i_{1}, j_{1}\right) \in H_{m}$. The dominance inequalities are defined as

$$
y_{i_{1} j_{1}}+y_{i_{2} j_{2}} \leq 1
$$

for any pair of $\operatorname{arcs}\left(i_{1}, j_{1}\right),\left(i_{2}, j_{2}\right) \in A_{R}$ such that $\left(i_{1}, j_{1}\right)$ dominates $\left(i_{2}, j_{2}\right)$ or viceversa. Note that if $\left(i_{1}, j_{1}\right)$ dominates $\left(i_{2}, j_{2}\right)$, the tours that service arc $\left(i_{1}, j_{1}\right)$, and thus satisfy $y_{i_{1} j_{1}}=1$, do not need to service arc $\left(i_{2}, j_{2}\right)$ (and thus we can set $y_{i_{2} j_{2}}=0$ ). Note also that, since there can be tours with $y_{i_{1} j_{1}}=1$ and $y_{i_{2} j_{2}}=1$, these inequalities are not valid for $\operatorname{GDRPP}(G)$, but there is always at least one optimal tour that satisfies them.

\section{A different formulation for the GDRPP}

In the formulation used in the previous sections, we distinguish between merely traversing an $\operatorname{arc}\left(x_{i j} \geq 1\right.$ and $\left.y_{i j}=0\right)$ and servicing a customer while traversing the $\operatorname{arc}\left(x_{i j} \geq 1\right.$ and $\left.y_{i j}=1\right)$. In some situations, the knowledge of whether a customer is being serviced or not from a given arc that is traversed by the solution may not be relevant. In this case, we could formulate the problem without using the $y_{i j}$ variables, as we will see in what follows. A formulation that does not use variables $y_{i j}$ could be interesting, mainly in situations in which the number of required arcs is high, because it would reduce the number of variables used from $|A|+\left|A_{R}\right|$ to $|A|$. 
In the following formulation, we associate one variable $x_{i j}$ with each arc, representing the number of times it is traversed:

$$
\begin{aligned}
& \sum_{(i, j) \in A} c_{i j} x_{i j} \\
& \text { M.t. : } \quad x\left(\delta^{-}(i)\right), \quad \forall i \in V \\
& x\left(\delta^{+}(i)\right) \\
& x\left(\delta^{+}(S)\right) \geq 1-x\left(H_{m} \cap A(V \backslash S)\right), \quad \forall S \subseteq V \backslash\{1\}, \forall H_{m} \in \mathbb{H} \\
& \sum_{(i, j) \in H_{m}} x_{i j} \geq 1, \quad \forall H_{m} \in \mathbb{H} \\
& x_{i j} \geq 0, \quad \forall(i, j) \in A \\
& x_{i j} \text { integer, } \quad \forall(i, j) \in A
\end{aligned}
$$

With respect to the former formulation, the new inequalities are (17), which force the solution to be connected and connected to the depot, and (18), which ensure that all the customers will be serviced.

Moreover, parity inequalities (12) and K-C inequalities (14) can be adapted to this new formulation. With regard to the new parity inequalities, note that replacing all the $y$ variables in the inequality by the corresponding $x$ variables may lead to non-valid inequalities, because $x$ variables are not binary. Therefore, we can only replace those $y$ variables that have negative coefficient in the RHS of the inequality and take $F=\emptyset$. So the new parity inequalities are written as

$$
x(\delta(S)) \geq \sum_{i=1}^{q}\left(1-2 x\left(H_{n_{i}} \backslash \delta(S)\right)\right)+1,
$$

where $H_{n_{1}}, H_{n_{2}}, \ldots, H_{n_{q}}$, with $q$ odd, satisfy:

- $H_{n_{i}} \cap H_{n_{j}} \cap \delta(S)=\emptyset \quad \forall n_{i}, n_{j}$ and

- $H_{n_{i}} \cap \delta(S) \neq \emptyset \quad \forall n_{i}$.

In the same way, we can adapt the $\mathrm{K}-\mathrm{C}$ inequalities to the new formulation by taking $F=\emptyset$ and $I_{2}=\emptyset$ (and hence $I_{1}=\{1, \ldots, K-1\}$ ). The new $\mathrm{K}$-C inequality is then

$$
\begin{gathered}
(K-2)\left(x\left(M_{0}: M_{K}\right)+x\left(M_{K}: M_{0}\right)\right)+\sum_{\substack{0 \leq i, j \leq K \\
(i, j) \neq(0, K)}}|i-j| x\left(M_{i}: M_{j}\right) \\
\geq(K-2) \sum_{i=1}^{q}\left(1-2 x\left(H_{n_{i}} \backslash A_{R}^{O K}\right)\right)+2 \sum_{j=1}^{K-1}\left(1-x\left(H_{m_{j}} \backslash A\left(M_{j}\right)\right)\right) .
\end{gathered}
$$

We would like to point out that inequalities (21) and (22) are just a direct adaptation of inequalities (12) and (14) developed for the former formulation. Therefore there may be stronger versions for the new formulation that have not yet been studied. 


\section{A branch-and-cut algorithm}

In this section we present a branch-and-cut algorithm based on the first formulation, which incorporates separation algorithms for the inequalities described in this paper.

\subsection{Separation algorithms}

In this section we present the separation algorithms that have been used to identify the inequalities violated by the current LP solution at any iteration of the cutting plane algorithm.

In order to separate $\mathrm{K}-\mathrm{C}, 2 \mathrm{~PB}$, and $\mathrm{A} 2 \mathrm{~PB}$ inequalities, we first apply a shrinking procedure to reduce the size of the support graph, which basically consists of shrinking all the non-required $\operatorname{arcs}(i, j)$ for which $x_{i j}=1$ or $x_{i j} \geq 2$. Then, heuristic procedures for separating K-C and $2 \mathrm{~PB}$ inequalities based on those presented in [3] for the Mixed GRP are applied. While applying the separation procedure for $2 \mathrm{~PB}$ inequalities, we also check for possible $\mathrm{A} 2 \mathrm{~PB}$-violated inequalities whenever we find an appropriate structure. As previously mentioned, the coefficients of all the arcs joining nodes in different paths, except the first, are given by the length of the shortest path using that first arc and those in the skeleton. We choose the arc with the largest value of $x$ in the solution as the first.

\subsubsection{Connectivity inequalities}

In order to identify violated connectivity inequalities we have designed a heuristic and an exact procedure.

In the heuristic procedure, given a fractional solution $\left(x^{*}, y^{*}\right)$, we first build the graph induced by the arcs $a$ such that $x_{a}^{*} \geq \varepsilon$, where $\varepsilon$ is a given parameter. If the support graph is not weakly connected, the connectivity inequalities associated with its weakly connected components $C_{1}, \ldots, C_{q}$ are checked for violation. For each $C_{i}$ containing at least one required arc $a$ with $y_{a}^{*}>\varepsilon$, if there is a set $H_{m}$ such that $H_{m} \subseteq \delta\left(C_{i}\right) \cup A\left(C_{i}\right)$, we check whether the inequality $x\left(\delta^{+}\left(C_{i}\right)\right) \geq 1$ is violated. If there is no such set

- we look for the $\operatorname{arc} a_{\max } \in A\left(C_{i}\right)$ with maximum $y^{*}$ value, $y_{\max }$, and check the inequality $x\left(\delta^{+}\left(C_{i}\right)\right) \geq y_{\max }$, and

- we look for the set $H_{m}$ such that $y^{*}\left(H_{m} \cap A\left(V \backslash C_{i}\right)\right)$ is minimum and check whether the inequality $x\left(\delta^{+}\left(C_{i}\right)\right) \geq 1-y\left(H_{m} \cap A\left(V \backslash C_{i}\right)\right)$ is violated.

The exact procedure consists of solving a maximum flow problem between the depot and each required arc in order to obtain minimum weight cutsets that may violate connectivity inequalities. Once a minimum weight cutset has been found, we check the different connectivity inequalities for violation as before.

\subsubsection{Parity inequalities}

We do not know whether parity inequalities (12) can be separated exactly in polynomial time. We have developed a heuristic algorithm which works as follows.

Given a fractional solution $\left(x^{*}, y^{*}\right)$, we build the graph induced by the $\operatorname{arcs}$ satisfying $x_{a}^{*}-y_{a}^{*} \geq$ $\varepsilon$ in the solution, if $a$ is required, and $x_{a}^{*} \geq \varepsilon$ otherwise. Let $C_{1}, \ldots, C_{k}$ be its weakly connected components. We study the components $C_{i}$ for which $y^{*}\left(\delta\left(C_{i}\right)\right)$ is close to an odd number, that is, 
$2 n+0.75 \leq y^{*}\left(\delta\left(C_{i}\right)\right) \leq 2 n+1.25$. We define set $F$ as the set of arcs $a \in \delta\left(C_{i}\right)$ such that $y_{a}^{*} \approx 1$. Then we look for $q=2 n+1-|F|$ sets $H_{m}$ satisfying the conditions expressed in subsection 3.3. This is done by iteratively choosing the set with minimum $y^{*}\left(H_{c_{i}} \backslash \delta\left(C_{i}\right)\right)$. Finally the parity inequality obtained is checked for violation.

\subsubsection{K-C inequalities}

The heuristic algorithm used for separating $\mathrm{K}-\mathrm{C}$ inequalities is based on the one proposed for the Mixed GRP in [3]. Given a fractional solution $\left(x^{*}, y^{*}\right)$, we consider the arcs with $y_{a}^{*} \geq \varepsilon$ as required arcs, which define the $R$-sets. For each $R$-set, we try to split it into $M_{0}$ and $M_{K}$ in such a way that $y^{*}\left(A_{R}^{0 K}\right)$ is close to an even value. Then we look for $\operatorname{arcs}$ to define set $F$ and sets $H_{m}$ to define $F^{H}$ in a similar way to that described for the parity inequalities. Finally, the $\mathrm{K}-\mathrm{C}$ inequality obtained is checked for violation.

\subsection{Initial relaxation and cutting-plane algorithm}

The initial LP relaxation contains all the inequalities in the formulation, except for the connectivity inequalities (3) and (4), of which only inequalities (3) associated with $S=H_{m}, \forall H_{m} \in \mathbb{H}$, and $S=V \backslash\{1\}$ are included. Furthermore, dominance inequalities (15) are also included.

At each iteration of the cutting plane algorithm the separation procedures are used in the following specific order and the violated inequalities found are added to the LP relaxation:

1. Heuristic separation algorithm for connectivity inequalities with $\varepsilon=0,0.25,0.5,0.75$.

2. Exact connectivity separation if the heuristic has failed to find violated inequalities and only at the root node.

3. Heuristic parity separation with $\varepsilon=0,0.25,0.5$.

4. Only at the root node, if no violated connectivity inequalities with $\varepsilon=0$ have been found, heuristic algorithm for separating $\mathrm{K}-\mathrm{C}$ inequalities.

The cutting-plane procedure is applied at each node of the tree until no new violated inequalities are found or a stopping criterion, called tailing-off, is satisfied. At the root node, the cutting plane stops when the increase in the objective function during the last 20 iterations is less than $0.001 \%$. At any other node, the cutting plane stops if the increase is less than $0.005 \%$ in the last five iterations.

\subsection{Upper bound}

We have implemented a heuristic algorithm to obtain feasible solutions using the fractional solution of the LPs at the nodes of the branch-and-cut tree. This heuristic consists basically of solving a Set Covering Problem to select a set of arcs $A^{\prime}$ covering all the customers and then solving a DGRP with the arcs in $A^{\prime}$ as required arcs and the depot as a required vertex.

Let us consider a fractional solution $\left(x^{*}, y^{*}\right)$. Required arcs are ordered according to their $y^{*}$ value. The arcs are iteratively selected starting with the one with the highest $y^{*}$ value to obtain a set $A^{\prime}$ servicing all the customers. In the event of a tie, the number of customers serviced by the arcs and not yet serviced by arcs already in $A^{\prime}$ is considered, as well as the indegree and the outdegree of the end nodes of the arcs according to the arcs already selected. If an arc does not service any customer not serviced by the previously selected arcs, it is ignored. Once a set $A^{\prime}$ 
covering all the customers has been obtained, a minimum cost tour traversing all the $\operatorname{arcs}$ in $A^{\prime}$ and visiting the depot is obtained using the exact algorithm for the DGRP described in [1].

This algorithm is executed every 100 iterations of the cutting-plane procedure at the root node. After the root node has been studied, it is executed every 20 nodes up to node number 200, every 50 nodes between nodes 201 and 500, and every 200 nodes beyond that.

\section{Computational experiments}

In this section we describe the GDRPP instances we have used and the results obtained on them with our branch-and-cut algorithm.

The algorithm was coded in $\mathrm{C}++$ using the Cplex 12.4 MIP Solver with Concert Technology 2.9 on a single thread of an Intel Core i7 at $3.4 \mathrm{GHz}$ with $16 \mathrm{~GB}$ of RAM. Cplex heuristic algorithms were turned off, while Cplex own cuts, including zero-half cuts, were activated in automatic mode. The optimality gap tolerance was set to zero, and strong branching and the best bound strategies were selected. Finally, in order to remove non-binding cuts, Cplex presolve phase was reapplied at the end of the root node, allowing for new iterations of the cutting-plane procedure before branching. All the tests were run with a time limit of two hours.

In order to compare the performance of our algorithm with the branch-and-cut procedure proposed by Hà et al. [10], we have used, where possible, the same sets of instances. Since we have not been able to reproduce their third set of instances, defined on undirected graphs, we have generated a new set of instances in which the customers define disjoint arc sets. These new instances and the best solutions found for all the sets can be found at http://www.uv.es/corberan/instancias.

The first set of instances generated by Hà et al. contains random instances based on directed graphs. Their characteristics are shown in Table 1. All these instances have 500 vertices and up to $1500 \mathrm{arcs}$, and the number of customers varies between 500 and 15000. Arc costs correspond to Euclidean distances multiplied by five to obtain an average arc length close to that seen in practice (from about 0.2 to $0.4 \mathrm{~km}$ ). We would like to point out that, contrary to usual practice, these costs have not been rounded to integer numbers but to numbers with up to 4 decimal places. Customers are positioned randomly in the square containing the graph and the arcs located closer than a given distance $r=150,200$ define the sets $H_{m}$. There are five different instances in each set. For example, the instance set named ce200-0.5 contains instances with $r=200$ and $t=0.5$, where $t$ is the ratio between the number of customers and the number of arcs.

The results obtained with our branch-and-cut algorithm on these instances are also shown in this table. Column GapO gives the average percentage gap between the lower bound at the root node and the optimal solution, if known, or the best solution found. Solved reports the number of instances solved to optimality, while columns Nodes and Time show the average number of nodes of the branch-and-cut tree and the average computing time (in seconds), respectively. The last three columns report the average number of violated connectivity, parity, and K-C inequalities found.

From Table 1, it can be seen that our algorithm is able to solve all the instances optimally in relatively small computing times. Since the gap at the root node is very small in all cases, we believe that the success of the algorithm is due to the tightness of the lower bound provided by the cutting-plane algorithm, which indicates that the new connectivity, parity, and K-C inequalities are useful for describing the polyhedron of solutions more accurately. Note also that the difficulty of these instances seems to decrease when the number of customers and the 
distance $r$ increase.

\begin{tabular}{lcccccrrrrr} 
& $|V|$ & $|A|$ & $|\mathbb{H}|$ & Gap0 & Solved & Nodes & Time & Conn. & Parity & K-C \\
\hline \hline ce200-0.5 & 500 & 1000 & 500 & 0,65 & $5 / 5$ & 148,8 & 245,7 & 9892,0 & 1311,6 & 2,8 \\
ce200-1 & 500 & 1000 & 1000 & 0,28 & $5 / 5$ & 47,8 & 88,6 & 4985,0 & 997,8 & 24,0 \\
ce200-5 & 500 & 1000 & 5000 & 0,08 & $5 / 5$ & 16,8 & 28,3 & 1093,0 & 668,0 & 6,8 \\
ce200-10 & 500 & 1000 & 10000 & 0,11 & $5 / 5$ & 12,2 & 20,3 & 429,8 & 583,4 & 3,0 \\
\hline ce150-0.5 & 500 & 1500 & 750 & 0,49 & $5 / 5$ & 1025,8 & 830,5 & 8118,2 & 2405,2 & 39,4 \\
ce150-1 & 500 & 1500 & 1500 & 0,38 & $5 / 5$ & 1924,2 & 1235,5 & 4871,8 & 1897,0 & 23,2 \\
ce150-5 & 500 & 1500 & 7500 & 0,12 & $5 / 5$ & 30,8 & 49,2 & 403,4 & 745,8 & 6,2 \\
ce150-10 & 500 & 1500 & 15000 & 0,13 & $5 / 5$ & 43,2 & 50,1 & 412,2 & 734,6 & 3,6 \\
\hline \hline
\end{tabular}

Table 1: Results on the "ce" instances

In Table 2 the results obtained with the branch-and-cut algorithm by Hà et al. [10] are compared with those obtained with ours. The Hà et al. algorithm has been run on a $2.4 \mathrm{GHz}$ CPU with $6 \mathrm{~GB}$ of RAM with a time limit of two hours. It uses Cplex 11.2 with all Cplex cuts turned off except the zero-half cuts, which are generated using the aggressive option. It also uses an initial upper bound provided by a heuristic described in the same paper. Column LBO-Imp shows the average improvement in percentage between the lower bounds of both algorithms at the end of the root node, i.e., $\frac{L B_{A}-L B_{H}}{L B} \times 100$, where $L B_{A}$ and $L B_{H}$ are the lower bounds at the root node obtained with the algorithm proposed here and the one by Hà et al., respectively. It can be seen that our algorithm produces better lower bounds and is able to solve more instances in shorter computing times.

\begin{tabular}{lrrrrrrr} 
& \multicolumn{2}{c}{ Hà et al. } & & \multicolumn{3}{c}{ Ávila et al. } \\
\cline { 2 - 3 } \cline { 5 - 7 } \cline { 5 - 6 } & Solved & Time & & Solved & Time & LB0-Imp \\
\hline \hline ce200-0.5 & 3 & 4155,6 & & 5 & 245,7 & 2,54 \\
ce200-1 & 4 & 2447,8 & & 5 & 88,6 & 2,11 \\
ce200-5 & 5 & 315,1 & & 5 & 28,3 & 0,87 \\
ce200-10 & 5 & 82,3 & & 5 & 20,3 & 0,79 \\
\hline ce150-0.5 & 0 & 7202,9 & & 5 & 830,5 & 2,04 \\
ce150-1 & 2 & 4499,9 & & 5 & 1235,5 & 1,43 \\
ce150-5 & 5 & 154,5 & & 5 & 49,2 & 0,47 \\
ce150-10 & 5 & 205,9 & & 5 & 50,1 & 0,26 \\
\hline \hline
\end{tabular}

Table 2: Comparison with the Hà et al. [10] results on the "ce" instances

We have also checked our algorithm on the second set of instances used in Hà et al. [10]. These are instances generated from two CPP instances, MB537 and MB547, defined on mixed graphs and proposed by Corberán et al. [4]. These graphs have a structure similar to that of real street networks and have the following characteristics. The mixed graph MB357 has 500 vertices, 364 edges and 476 arcs, while graph MB547 has 500 vertices, 351 edges and 681 arcs. The mixed graphs are transformed into directed ones by replacing each edge with two parallel arcs with the same cost. The procedure to generate the customers is the same as the one used for the "ce" instances, except that the distance parameter $r$ is determined by the average length of all the arcs in the graph. For each graph and each value of $t$, Hà et al. generated five GDRPP instances.

Table 3 shows the results obtained with our branch-and-cut algorithm on these GDRPP instances. All the instances except one have been solved to optimality. As with the "ce" instances, the gap values at the root node are small and the computing times are reasonable. 
The comparison between the results obtained with the branch-and-cut by Hà et al. [10] and with our algorithm are shown in Table 4. Note that our algorithm finds more optimal solutions in shorter computing times and usually produces better lower bounds, except on the instances with the largest number of customers.

\begin{tabular}{lcccccrr} 
& $|V|$ & $|A|$ & $|\mathbb{H}|$ & Gap0 & Solved & Nodes & Time \\
\hline \hline MB0537-0.5 & 500 & 1204 & 400 & 0,16 & $5 / 5$ & 14,6 & 456,3 \\
MB0537-1 & 500 & 1204 & 800 & 0,20 & $5 / 5$ & 36,8 & 368,5 \\
MB0537-5 & 500 & 1204 & 4000 & 0,19 & $5 / 5$ & 34,0 & 223,8 \\
MB0537-10 & 500 & 1204 & 8000 & 0,18 & $5 / 5$ & 73,8 & 233,4 \\
\hline MB0547-0.5 & 500 & 1383 & 520 & 1,17 & $4 / 5$ & 2685,8 & 2854,3 \\
MB0547-1 & 500 & 1383 & 1040 & 0,78 & $5 / 5$ & 1297,6 & 1515,2 \\
MB0547-5 & 500 & 1383 & 5200 & 0,19 & $5 / 5$ & 176,8 & 232,1 \\
MB0547-10 & 500 & 1383 & 10400 & 0,18 & $5 / 5$ & 45,4 & 131,5 \\
\hline \hline
\end{tabular}

Table 3: Results on the "MB" instances

\begin{tabular}{lrrrrrrr} 
& \multicolumn{2}{c}{ Hà et al. } & & \multicolumn{3}{c}{ Ávila et al. } \\
\cline { 2 - 3 } \cline { 5 - 7 } & Solved & Time & & Solved & Time & LB0-Imp \\
\hline \hline MB0537-0.5 & 0 & 7200,7 & & 5 & 456,3 & 5,7 \\
MB0537-1 & 0 & 7201,5 & & 5 & 368,5 & 5,7 \\
MB0537-5 & 3 & 3937,8 & & 5 & 223,8 & 0 \\
MB0537-10 & 5 & 2418,3 & & 5 & 233,4 & -1 \\
\hline MB0547-0.5 & 0 & 7201,1 & & 4 & 2854,3 & 5 \\
MB0547-1 & 0 & 7202,2 & & 5 & 1515,2 & 3,5 \\
MB0547-5 & 4 & 1639,9 & & 5 & 232,1 & $-0,3$ \\
MB0547-10 & 5 & 756,2 & & 5 & 131,5 & -1 \\
\hline \hline
\end{tabular}

Table 4: Comparison with the Hà et al. [10] results on the "MB" instances

The third set of instances has been generated from undirected RPP instances used in Corberán, Plana and Sanchis [5]. Again, the underlying undirected graphs are transformed into directed ones by replacing each edge by a pair of opposite arcs with the same cost. For these instances, the family of arc subsets $\mathbb{H}=\left\{H_{1}, \ldots, H_{L}\right\}$ has been generated in a different way: the arcs corresponding to each connected component induced by the required edges in the original graph define the sets $H_{m}$. Note that, in this case, the sets $H_{m}$ are disjoint. The instances called UR500 have $298 \leq|V| \leq 499,597 \leq|A| \leq 1526$, and $1 \leq|\mathbb{H}| \leq 99$. UR750 instances have $452 \leq|V| \leq 749,915 \leq|A| \leq 2314$, and $1 \leq|\mathbb{H}| \leq 140$. Finally, UR1000 instances have $605 \leq|V| \leq 1000,2289 \leq|A| \leq 3083$, and $1 \leq|\mathbb{H}| \leq 204$. There are 12 GDRPP instances in each group.

The average characteristics of each group of instances are shown in Table 5, as well as the average results obtained on them with the proposed branch-and-cut. The algorithm is able to solve to optimality all 12 instances in the set UR500 and 10 out of 12 instances in UR750, but only two instances in the UR1000 set. In fact, in one of the UR1000 instances, the algorithm could not even find a feasible solution in the time limit of two hours. Even so, the gap at the root node (with respect to the optimum or the best solution found) and the final gap are small in 


\begin{tabular}{lcccrrrrr} 
& $|V|$ & $|A|$ & $|\mathbb{H}|$ & Gap0 & Gap & Solved & Nodes & Time \\
\hline \hline UR500 & 446,0 & 2257,8 & 35,3 & 0,24 & 0,00 & $12 / 12$ & 320,2 & 790,0 \\
UR750 & 665,7 & 3396,8 & 55,7 & 0,32 & 1,18 & $10 / 12$ & 1590,0 & 3247,0 \\
UR1000 & 882,2 & 4580,8 & 74,8 & 3,58 & 4,34 & $2 / 12$ & 1262,0 & 6016,3 \\
\hline \hline
\end{tabular}

Table 5: Results on the "UR" instances (in which sets $H_{m}$ are disjoint)

most instances. Regarding the computing times, it seems that the fact that the customers define disjoint sets affects the difficulty of the problem. It should be noted, however, that the algorithm and the separation procedures had not considered this possibility, as they were designed to solve instances where customers could have common elements. The same is true for the upper bound. The heuristic described in Section 5.3 did not work well in this case, so the upper bound provided was of no help in solving these instances. In our opinion, a more appropriate implementation of the heuristic and the branch-and-cut algorithm for the case of disjoint customers could lead to better results in this case.

Finally, in order to test the potential of the second formulation for the GDRPP presented in Section 4, we have adapted the branch-and-cut algorithm to work only with " $x$ " variables and checked it on the "ce" instances. The results obtained are compared in Table 6 with those obtained with the original algorithm (already shown in Table 1). It can be seen that the performance of the algorithm based on the second formulation is much worse. That said, we want to point out that the possibilities of the new formulation have not been explored in depth. Not only may there be stronger versions of the inequalities for the second formulation, as we said in Section 4, but also a better implementation of the separation routines and the heuristic algorithm could produce better computational results.

\begin{tabular}{|c|c|c|c|c|c|c|c|c|c|}
\hline & \multirow[b]{2}{*}{$|V|$} & \multirow[b]{2}{*}{$|A|$} & \multirow[b]{2}{*}{$|\mathbb{H}|$} & \multicolumn{3}{|c|}{ First formulation } & \multicolumn{3}{|c|}{ Second formulation } \\
\hline & & & & Solved & Time & Gap0 & Solved & Time & Gap0 \\
\hline ce200-0,5 & 500 & 1000 & 500 & $5 / 5$ & 245,7 & 0,65 & $5 / 5$ & 1079,92 & 1,81 \\
\hline ce200-1 & 500 & 1000 & 1000 & $5 / 5$ & 88,6 & 0,28 & $5 / 5$ & 856,48 & 1,41 \\
\hline ce200-5 & 500 & 1000 & 5000 & $5 / 5$ & 28,3 & 0,08 & $5 / 5$ & 187,96 & 0,82 \\
\hline ce200-10 & 500 & 1000 & 10000 & $5 / 5$ & 20,3 & 0,11 & $5 / 5$ & 137,48 & 1,17 \\
\hline ce150-0,5 & 500 & 1500 & 750 & $5 / 5$ & 830,5 & 0,49 & $1 / 5$ & 6025,43 & 2,74 \\
\hline ce150-1 & 500 & 1500 & 1500 & $5 / 5$ & 1235,5 & 0,38 & $2 / 5$ & 4036,92 & 2,32 \\
\hline ce150-5 & 500 & 1500 & 7500 & $5 / 5$ & 49,2 & 0,12 & $5 / 5$ & 331,83 & 0,99 \\
\hline ce150-10 & 500 & 1500 & 15000 & $5 / 5$ & 50,1 & 0,13 & $4 / 5$ & 1739,64 & 1,26 \\
\hline
\end{tabular}

Table 6: Comparison between the two GDRPP formulations

\section{Conclusions}

In this paper we have addressed the polyhedral description and the resolution of the Generalized Directed Rural Postman Problem (GDRPP). We have proposed a new formulation and presented some new families of valid inequalities. On the basis of this description, we have implemented a branch-and-cut algorithm for the GDRPP and provided extensive computational experiments over various sets of GDRPP instances. The results show that our algorithm outperforms the existing exact methods. We have also introduced an alternative formulation with fewer variables whose study could be of interest in the future. 
Acknowledgements: The authors wish to thank Minh Hoang Hà, Nathalie Bostel, André Langevin, and Louis-Martin Rousseau for providing us with their instances. We also thank the Spanish Ministerio de Economía y Competitividad (project MTM2012-36163-C06-02) and the Generalitat Valenciana (project GVPROMETEO2013-049) for their support.

\section{References}

[1] T. Ávila, Á. Corberán, I. Plana, and J.M. Sanchis. The stacker crane problem and the directed general routing problem. Networks, to appear, 2014.

[2] E. Balas and M. Ng. On the set covering polytope I: All the facets with coefficients in \{0,1,2\}. Mathematical Programming, 43:57-69, 1989.

[3] Á. Corberán, G. Mejía, and J.M. Sanchis. New results on the mixed general routing problem. Operations Research, 53:363-376, 2005.

[4] Á. Corberán, E. Mota, and J.M. Sanchis. A comparison of two different formulations for arc routing problems on mixed graphs. Computers \& Operations Research, 33:3384-3402, 2006 .

[5] Á. Corberán, I. Plana, and J.M. Sanchis. A branch \& cut algorithm for the windy general routing problem and special cases. Networks, 49:245-257, 2007.

[6] Á. Corberán and J.M. Sanchis. A polyhedral approach to the rural postman problem. European Journal of Operational Research, 79:95-114, 1994.

[7] M. Drexl. On some generalized routing problems. PhD thesis, Rheinisch-Westfälische Technische Hochschule, Aachen University, 2007.

[8] M. Drexl. On the generalized directed rural postman problem. Journal of the Operational Research Society, 65:1143-1154, 2014.

[9] M.-H. Hà, N. Bostel, A. Langevin, and L.-M. Rousseau. An exact algorithm for close enough traveling salesman problem. In Proceedings of the 1st International Conference on Operations Research and Enterprise Systems, pages 233-238, 2012.

[10] M.-H. Hà, N. Bostel, A. Langevin, and L.-M. Rousseau. Solving the close enough arc routing problem. Networks, 63:107-118, 2014.

[11] R. Shuttleworth, B.L. Golden, S. Smith, and E. Wasil. Advances in meter reading: Heuristic solution of the close enough traveling salesman problem over a street network. In B. Golden, S. Raghavan, and E. Wasil, editors, The Vehicle Routing Problem: Lastest Advances and New Challenges, pages 487-501. Springer, 2008. 